\title{
Dermatofitoses podais em futebolistas
}

\author{
Feet dermatophytosis in Soccer Players
}

\author{
Kátia Sheylla Malta Purim ${ }^{1}$ \\ Neiva Leite ${ }^{3}$
}

\author{
Camila Fernanda Novak Pinheiro de Freitas ${ }^{2}$
}

\begin{abstract}
Resumo: Atletas apresentam risco para micoses cutâneas. Estudados 23 jogadores de futebol, através de exames clínico, micológicos (direto e cultura) e clipping ungueal, dezoito $(78,26 \%)$ não apresentavam micoses; dois $(8,70 \%)$ apresentavam tinea pedis e três $(13,04 \%)$ onicomicose, associada à tinea pedis, principalmente por Trichophyton mentagrophytes. Tinea pedis infectada produziu celulite em um atleta. É necessário um programa educativo de cuidados com a pele no esporte.

Palavras-chave: Dermatoses do pé; Onicomicose; Tinha dos pés

Abstract: Athletes present risk of cutaneous mycosis. A study was carried out with 23 soccer players using clinical and mycological examination (direct microscopic examination and culture) and nail clipping. Eighteen (78.26\%) did not present mycosis; two (8.70\%) presented tinea pedis, and three (13.04\%) presented onychomycosis associated to tinea pedis, mainly for Trichophyton mentagrophytes. Infected tinea pedis has produced cellulitis in one of the athletes. It is necessary to create an educative program of skin care during sports practice.

Keywords: Foot dermatoses; Onychomycosis; Tinea pedis
\end{abstract}

\section{COMUNICAÇÃO}

A terminologia "pé-de-atleta" ou "frieira" é associada à prática desportiva, entretanto, são escassos, na literatura, estudos na população que exerce o esporte profissionalmente. No futebol, a tinea pedis, principalmente, quando infectada ou associada à onicomicose, ultrapassa o sujeito individual para refletir no coletivo, sendo fundamental a prevenção. ${ }^{1-4}$

Pesquisa transversal e descritiva, aprovada pelo Comitê de Ética do HC-UFPR, avaliou a prevalência de dermatofitoses podais em 23 jogadores masculinos de futebol profissional em Curitiba (PR), através de exames micológicos direto e culturas dos pés: unha, região plantar, interdigital e ainda, exame histopatológico de fragmento ungueal, corado pelo PAS com digestão. ${ }^{5}$
Os atletas apresentavam idade entre 18 a 30 anos (média 23,48 $\pm 3,06$ ), peso 75,57 $\pm 6,29 \mathrm{~kg}$, estatura $1,79 \pm 0,05$ metros, tempo de profissão 6,13 $\pm 2,77$ anos, sendo dez (43,48\%) brancos, nove $(39,13 \%)$ mulatos e quatro (17,39\%) negros. Procediam do sul $(39,13 \%)$ e sudeste $(21,74 \%)$ do país. Dezoito atletas $(78,26 \%)$ cursaram de quatro a oito anos de ensino regular; quatro atletas $(17,39 \%)$ possuíam ensino médio completo e um $(4,35 \%)$ cursava a Faculdade de Educação Física.

Três casos $(13,04 \%)$ apresentavam onicomicose, associada à tinea pedis, e dois $(8,70 \%)$, somente tinea pedis. Todos os casos de onicomicose estavam associados a tinea pedis interdigital. (Tabela 1)

Aprovado pelo Conselho Editorial e aceito para publicação em 18.12.2008.

* Trabalho realizado no Serviço de Dermatologia do Hospital de Clínicas da Universidade Federal do Paraná (UFPR) - Curitiba (PR), Brasil.

Conflito de interesse: Nenhum / Conflict of interest: None

Suporte financeiro: Nenhum / Financial funding: None

Dermatologista. Doutora em Medicina e Ciências da Saúde (UFPR). Professora Titular de Dermatologia da Universidade Positivo (UP). Professora colaboradora do Serviço de Dermatologia do Hospital de Clínicas da Universidade Federal do Paraná (UFPR) - Curitiba (PR), Brasil.

Acadêmica do quinto ano do Curso de Medicina da Universidade Federal do Paraná (UFPR) - Curitiba (PR), Brasil.

Médica do esporte e pediatra. Doutora em Saúde da Criança e do Adolescente (UFPR). Líder do Núcleo de Pesquisa em Qualidade de Vida (UFPR). Professora Coordenadora do Mestrado e Doutorado do Departamento de Educação Física da Universidade Federal do Paraná (UFPR) - Curitiba (PR), Brasil

(C)2009 by Anais Brasileiros de Dermatologia 
Tabela 1: Prevalência de dermatofitose na região dos pés de 23 atletas profissionais de futebol

Dermatofitose na região dos pés Atletas profissionais

\begin{tabular}{lll} 
& $(\mathrm{n}=23)$ & $\%$ \\
\hline Ausência de dermatofitose & 18 & 78,26 \\
Tinea pedis isolada & 2 & 8,70 \\
Onicomicose associada & 3 & 13,04 \\
à tinea pedis & & $\mathbf{1 0 0 , 0 0}$
\end{tabular}

TABela 2: Resultados dos exames obtidos no estudo de 23 atletas profissionais de futebol

\begin{tabular}{llll}
\hline Área / material & Exames laboratoriais & \multicolumn{2}{c}{$\begin{array}{c}\text { Atletas profissionais } \\
\text { (n=23) }\end{array}$} \\
\hline Região plantar & & 2 & $(9.1)$ \\
Região interdigital & Exame direto & - & - \\
& Cultura & 2 & 9.1 \\
Unha & Exame direto & 4 & 18.2 \\
Unha & Cultura & 1 & 4.6 \\
& Exame direto & 2 & 9.1 \\
\hline
\end{tabular}

Em dois casos (9,09\%), o exame micológico direto demonstrou dermatófito, de região plantar; em dois casos (9,09\%), de região interdigital do pé, e em um caso $(4,54 \%)$, na unha do pododáctilo. O exame histopatológico do clipping ungueal foi positivo, em dois casos (9,09\%). Em quatro casos (18,18\%), a cultura de região interdigital do pé foi positiva e, em dois casos (9,09\%), nas unhas dos pododáctilos, sendo que um dos atletas apresentou positividade na cultura da região interdigital e unha. (Tabela 2)

Neste estudo, o percentual de casos de tinea pedis $(21,74 \%)$ foi menor do que os resultados do Projeto Achilles brasileiro. ${ }^{6,7}$ Trichopbyton mentagrophytes (60\%) foi o principal agente etiológico, seguido do Trichophyton rubrum, em $20 \%$. Nos dois atletas (8,70\%) com tinea pedis, existiam históricos e diagnósticos prévios de micoses. Um atleta foi afastado de jogos por celulite de membro inferior, devido à tinea pedis infectada, gerando custos $\mathrm{e}$ adaptações individuais e coletivas. As trocas frequentes de calçados e equipamentos neste time, provavelmente, minimizaram as condições para desenvolvimento de fungos.
Nos três atletas (13,04\%), com onicomicose, associada à tinea pedis, o hálux foi o local mais comprometido, sendo este achado comum.,

Ocorreu maior positividade no exame direto $(66,7 \% \mathrm{p}=0,0344)$ e cultura $(66,7 \% \mathrm{p}=0,0119) \mathrm{de}$ região interdigital dos atletas que não secavam regularmente os pés, sendo Trichophyton mentagrophytes, o agente prevalente. ${ }^{7}$ Exame histológico da queratina ungueal complementou o estudo micológico, apresentando correlação nos resultados. ${ }^{5}$

Calos (95\%) e bolhas (26\%) chamavam atenção para um conjunto de atributos que poderia ser denominado "pé-do-atleta". As características clínicas dos pés dos atletas, notadamente, anatomo-funcionais (desvio de pododáctilos e hálux valgo), com as condições específicas do trabalho (gestos, posições movimentos, esforços, ritmos, fatores agressivos diversos), foram direta e indiretamente causadas, mantidas ou agravadas pela atividade profissional.

A limitação deste estudo está no pequeno número de casos. Investigações complementares podem elucidar melhor as relações das infecções fúngicas no futebol. 


\section{REFERÊNCIAS}

1. Caputo R, de Boule $K$, Del Rosso J, Novichi $R$. Prevalence of superficial fungal infections among sports-active individuals: results from the Achilles survey, a review of the literature. J Eur Acad Dermatol Venerol. 2001;15:312-6.

2. Purim KS, Bordignon GF, Queiroz-Telles F. Fungal infection of the feet in soccer players and non-athlete individuals. Rev Iberoam Micol. 2005;22:34-8.

3. Purim KSM, Niehues LP, Queiroz-Telles F, Leite N. Aspectos epidemiológicos das micoses dos pés em um time chinês de futebol. Rev Bras Med Esporte. 2006;22:16-20.

4. Pickup TL, Adams BB. Prevalence of tinea pedis in professional and college soccer players versus nonathletes. Clin J Sport Med. 2007;17:52-4.

5. Suarez SM, Silvers DN, Scher RK, Pearlstein $\mathrm{HH}$, Auerbach R. Histologic evaluation of nail clippings for diagnosing onychomycosis. Arch Dermatol. 1991; 127:1517-9.
6. Zaitz C. Projeto Achilles. An Bras Dermatol. 1999;74 (Suppl 2):S25-36.

7. Lacaz CS, Porto E, Martins JEC, Heins-Vaccari EM, Melo NT. Tratado de micologia médica. São Paulo: Sarvier; 2002. p. 252-352.

ENDEREÇO PARA CORRESPONDÊNCIA / MAILING ADDRESS:

Kátia Sbeylla Malta Purim

Hospital de Clínicas da UFPR

Serviço de Dermatologia

Rua General Carneiro, 180 - Centro

80060150 Curitiba, Paraná

Tel./Fax: +55(41) 33601800

kspurim@gmail.com

Como citar este artigo/How to cite this article: Purim KSM, Freitas CFNP, Leite N. Dermatofitoses podais em futebolistas. An Bras Dermatol. 2009;84(5):550-2. 